\title{
Governo eletrônico brasileiro: ações de integração entre sistemas de governo e sociedade
}

\author{
Gabriel da Silva Vieira ${ }^{1 *}$ \\ 1Departamento de Informática, Instituto Federal Goiano - Campus Urutaí, GO, Brasil. *Autor para correspondência: gabriel.vieira@ifgoiano.edu.br
}

\section{N F O A R T I G O}

Histórico do artigo

Recebido: 01 agosto 2015

Aceito: 22 novembro 2015

\section{Palavras chaves:}

Governo eletrônico

Interoperabilidade

e-PING

Tecnologia da informação e comunicação

\begin{abstract}
A B S T R A C T
Over the past decades, we have seen a proliferation of computer-based systems. Notably computer systems of different nature are present in daily life, in schools, businesses, and government, among others. The current challenge is to make such a large amount of systems become interoperable computer systems. In order to promote integration between government and society, the Brazilian federal government has been promoting actions so that this integration occurs. This paper investigates these solutions. Firstly, a literature review on e-government and interoperability is showed. After that, this work discusses Brazilian government's actions aimed at society. At this moment, technologies and forms of participation of the software development community in Brazilian egovernment were investigated too. This study also investigates integration initiatives with structuring systems of the federal government and applications developed by the community. It is concluded that the Brazilian federal government has a set of actions that effect their proposal for e-government. That the initiatives of the federal government could be better organized, following the taxonomy adopted by the literature. That web services, and technologies that compose it, appear as important actors to apply interoperability. And, that Brazilian government could disclose its electronic platform with more consistency and stimulate society in making integration actions with government data.
\end{abstract}

\section{Introdução}

Sistemas de informação têm auxiliado organizações dos mais variados portes a tomarem decisões sob o foco de seu negócio empresarial ou de atividade pública. No âmbito da administração pública, esforços têm sido desprendidos em função da necessidade de organização de dados para a geração de conhecimento e também para garantir que a informação governamental possa ser rapidamente localizada e transmitida entre os diversos órgãos de governo e sociedade.

Um marco na democratização do acesso à informação é a implantação do governo eletrônico brasileiro, no ano de 2000, através de um Grupo de Trabalho Interministerial (Brasil, 2000). A partir daí, várias ações foram tomadas pelo poder executivo federal para transformar a relação do governo com a sociedade e promover interatividade com cidadãos, empresas e órgãos governamentais.

Dessa forma, na medida em que o governo transpunha suas ações a sistemas de informação, em grande parte estimulada pelos impactos globais trazidos pelas novas tecnologias, demandas foram surgindo, destacando-se àquelas voltadas ao uso racional de recursos e iniciativas que garantissem a integração das ações dos vários níveis de governo, dos três poderes, bem como com parceiros, fornecedores e o cidadão.

Este trabalho investiga e discute as iniciativas implementadas pelo governo federal brasileiro na promoção de integração e interatividade de sistemas de governo com a sociedade. 0 presente artigo está organizado em cinco seções. Na primeira seção, apresenta-se a estrutura de pesquisa que direcionou este estudo. Nesse momento, o elemento motivador da pesquisa, questões problema, objetivos e metodologia são explicitados. Na segunda e terceira seções, é feito uma breve revisão de literatura que contextualiza o governo eletrônico e o conceito de interoperabilidade, respectivamente. Na quarta seção os resultados de pesquisa são reunidos e organizados em duas subseções. A primeira subseção relata algumas das ações que constituem o governo eletrônico brasileiro, ao mesmo tempo em que discute ações voltadas à sociedade. A segunda subseção traz algumas iniciativas realizadas por instituições de governo para a integração de sistemas, bem como algumas iniciativas implementadas pela sociedade que utilizam dados abertos. 
Finalmente, na última seção, algumas conclusões são apresentadas.

\section{Motivação e Organização da Pesquisa}

Segundo o sítio web oficial do governo eletrônico, no âmbito do poder executivo federal, (http://governoeletronico.gov.br/), as diretrizes que o compõem atuam em três frentes fundamentais. São elas: estar junto ao cidadão; promover a melhoria da sua própria gestão interna; e atuar na integração com parceiros e fornecedores.

Ainda segundo esse sítio, essas diretrizes gerais orientam as ações do governo para a promoção da participação e do controle social, para a interpretação da inclusão digital como direito de cidadania, promoção de software livre para a otimização de recursos e investimentos em tecnologia da informação (TI), compartilhamento de recursos entre órgãos públicos a fim de racionalizar os seus usos, e definição e publicação de políticas, padrões, normas e métodos para sustentar as ações de implantação e operação do governo eletrônico.

Logo, com base nessa composição de instruções, surgem alguns questionamentos. Dentre eles, quais são as iniciativas aplicadas no governo eletrônico brasileiro para atender as diretrizes que o orientam? Quais seriam os serviços do governo eletrônico projetadas para que a sociedade faça uso? E, qual a estratégia adotada pelo governo para direcionar a sociedade na utilização desses serviços? No âmbito técnico, outros questionamentos também surgem. Por exemplo, quais tecnologias são empregadas para a integração de sistemas de governo? E ainda, há iniciativas de integração desenvolvidas pela comunidade, através de soluções computacionais?

Dessa forma, frente as diretrizes apresentadas, cabe verificar quais ações foram, e estão sendo tomadas pelo governo federal brasileiro para efetivar práticas de cidadania, reúso e integração. Nesse sentido, o presente estudo buscou, inicialmente, caracterizar o papel do governo eletrônico, para logo em seguida, contextualizar a interoperabilidade como fator determinante para a efetivação de sistemas eletrônicos de governo. A partir das contextualizações realizadas, o estudo se direcionou a identificar e discutir as ações do governo eletrônico brasileiro, em especial àquelas destinadas à sociedade. Ao mesmo tempo, averiguou os padrões utilizados para a integração de sistemas de governo e soluções computacionais desenvolvidas pela comunidade para 0 consumo de dados abertos.

A estratégia metodológica adotada neste estudo constituiu-se da revisão de literatura sobre a temática em questão e estudo de caso com base em análise documental. Buscou-se, inicialmente, consultar a literatura a fim de definir e contextualizar o governo eletrônico, e, ainda, discutir o papel da interoperabilidade como esforço de governança. Logo depois, o estudo de caso, com base em consultas ao sítios web da administração pública federal, em 2015/1, foi aplicado com intuito de analisar e discutir as iniciativas do governo para a efetivação de práticas de governo eletrônico.

\section{Governo Eletrônico}

Os princípios que regem a administração pública, em conjunto com preceitos constitucionais democráticos, orientam que ela deve entregar, de forma qualitativa e eficiente, os serviços essenciais que atendam as necessidades dos cidadãos, fornecer oportunidades de crescimento econômico, bem como facilitar o envolvimento do cidadão e participação na elaboração de políticas públicas e de prestação de serviços, de modo a promover o bem comum. Nesse sentido, o governo eletrônico (e-government) pode providenciar oportunidades para transformar a administração pública em um instrumento de provimento de melhores serviços e responder às exigências de transparência e prestação de contas (accountability).

Segundo Tang \& Perumal (2013), governo eletrônico se caracteriza pelo uso de Tecnologias de Informação e Comunicação (TIC, incluindo a world wide web, dispositivos móveis, conteúdo digital, etc.) por parte do setor público para melhorar e aumentar a eficiência e a eficácia da prestação de informações e serviços aos cidadãos, empresas e organizações. Dessa forma, transformando a relação do governo com a sociedade, com o setor privado e com outros governos, de uma forma mais interativa e colaborativa.

Nessa mesma linha de raciocínio, para o Banco Mundial (www.worldbank.org), governo eletrônico "refere-se ao uso, por agências governamentais, de tecnologias de informação (como redes de longa distância, internet e computação móvel) capazes de transformar as relações com cidadãos, empresas e outras unidades do governo".

Para Crowley (2008), na medida em que as TIC avançam, sistemas de governo eletrônico aumentam, são melhorados e passam a atingir melhor os seus objetivos.

Segundo Shailendra et al. (2007), as definições de governo eletrônico em um conjunto variado de fontes convergem para um tema em comum, o uso de tecnologia da informação, em especial a internet para melhorar a prestação de serviços do governo para cidadãos, empresas e outros órgãos governamentais.

Todavia, Patnaik et al. (2008) alerta que apenas o uso de tecnologias não garante um governo eletrônico efetivo, sendo igualmente importante o envolvimento de pessoas e gestão de processos. Santos \& Reinhard (2008) ampliam esse entendimento ao apontar que o conceito de governo eletrônico encontra-se na intersecção de três áreas do conhecimento: políticas públicas, gestão e comportamento organizacional, e tecnologia da informação.

Uma interpretação seccionada sobre esse tema ocorre quando se classifica o governo eletrônico baseado no relacionamento dele com os interessados em seus serviços, como cidadãos, empresas, outros órgãos governamentais, e funcionários do próprio governo.

Surgem então, quatro tipos de transações: G2G (government to government), lida com as atividades que ocorrem entre as diferentes organizações governamentais; G2B (governments to business), caracterizado por transações entre governos e pessoas jurídicas em seus vários papéis desempenhados; G2C (government to citizens), atividades em que o governo fornece acesso online a informações e serviços ao cidadão; e G2E (government to employee), relacionadas às transações entre governos e seus próprios servidores (Shailendra et al. 2007, Patnaik et al. 2008, Santos \& Reinhard, 2008, Obeidat \& Abu-Shanab, 2010, Alshehri \& Drew, 2011).

Outros vocábulos que se relacionam ao tema governo eletrônico são: e-governance, e-democracy, m-government e $e$ participation (Tang \& Perumal, 2013, Nações Unidas, 2014).

Indo além de uma definição, governos de vários países do mundo, desenvolvidos e em desenvolvimento, estão em processo de uso de novas tecnologias, particularmente ferramentas ligadas à internet, para promover a entrega de serviços integrados aos cidadãos, tais como, pagamento de contas, informações sobre serviços do governo e registros autenticados de documentos.

0 governo italiano, por exemplo, possui um portal ${ }^{1}$ online que dá aos cidadãos a possibilidade de analisar e monitorar, em tempo real, a implementação de requisitos de dados e de informação impostas pela lei italiana sobre os sites da administração pública. 0 núcleo do sistema é um motor que, por meio de vários sensores de software e algoritmos matemáticos, analisa automaticamente os sites em tempo real ou periodicamente. 0 motor verifica a presença do conteúdo 
que deve ser legalmente publicado na página inicial e nas páginas internas de mais de 10.000 sítios web da administração.

Outro exemplo é a plataforma de e-government do governo uruguaio ${ }^{2}$. Com o objetivo geral de possibilitar e promover o desenvolvimento de serviços de governo eletrônico no país, essa plataforma segue uma abordagem em duas frentes. A primeira consiste em uma plataforma de interoperabilidade e a segunda em um conjunto de serviços transversais. Essa plataforma implementa uma arquitetura orientada a serviços com uso de web services para expor, utilizar e combinar funcionalidades implementadas por órgãos públicos.

Já o governo brasileiro lançou a partir do ano de 2000, no âmbito do poder executivo federal, um conjunto de políticas, diretrizes e normas relacionadas às novas formas eletrônicas de interação (Brasil, 2000).

Segundo Tonhati (2011), o processo de introdução da gestão pela qualidade iniciada nos anos 90, no Brasil, encontrou no governo eletrônico o modo de operacionalizar e por em prática as transformações almejadas para promover a modernização da administração.

Laia (2011) complementa ao afirmar que os movimentos de reforma do Estado influenciaram o surgimento do governo eletrônico em 2000, que herdou características e estruturas de utilização de TIC da administração pública brasileira.

Diniz et al. (2009) afirma que a história do governo eletrônico no Brasil está diretamente ligada ao desenvolvimento dos serviços de informática pública, oferecidos pelas empresas estaduais e municipais de processamento de dados, iniciado nos primeiros anos da década de 1960.

Antes da ideia e conceito de governo eletrônico ser formalmente criado na década de 1990 (Misra, 2006), muitos governos já haviam iniciado suas próprias iniciativas e programas com o uso de TIC (Tang \& Perumal, 2013). Apesar disso, o grau de desenvolvimento em governo eletrônico varia consideravelmente de um país a outro, independente de pioneirismo.

De acordo com o relatório "E-Government Survey 2014: e-government for the future we want" das Nações Unidas, entre os dez países que lideram o ranking mundial em aplicação de e-government estão nas cinco primeiras posições, a República da Coreia, Austrália, Singapura, França e Holanda, respectivamente. 0 quadro 1 apresenta os principais líderes em e-government segundo relatório supracitado.

Quadro 1. Líderes mundiais em e-government (Nações Unidas, 2014).

\begin{tabular}{ccc}
\hline Posição & País & Continente \\
\hline 1 & República da Coreia & Ásia \\
\hline 2 & Austrália & Oceania \\
\hline 3 & Singapura & Ásia \\
\hline 4 & França & Europa \\
\hline 5 & Holanda & Europa \\
\hline 6 & Japão & Ásia \\
\hline 7 & Estados Unidos da América & América \\
\hline 8 & Reino Unido & Europa \\
\hline 9 & Nova Zelândia & Oceania \\
\hline 10 & Finlândia & Europa \\
\hline
\end{tabular}

Desde 2003, o framework das Nações Unidas intitulado United Nations E-Government Survey adota uma visão holística do desenvolvimento de governo eletrônico pautado em três dimensões: (1) disponibilidade de serviços online , (2) infraestrutura de telecomunicação e (3) capacitação humana (Nações Unidas, 2014).

Com base nesse framework, as Nações Unidas indicam o progresso de seus países membros (193 países em 2014) através de índices para cada uma dessas dimensões. 0 cruzamento de dados entre esses índices formam então o $E$ Government Development Index (EGDI). A figura 1 ilustra as médias continentais em comparação com a média mundial de 0.4712 (EGDI). Em 2014, a Europa liderou com 0.6936 de EGDI, seguido pelas Américas com 0.5074, Ásia com 0.4951, Oceania com 0.4086 e finalmente Africa com 0.2661 .

Entre os países membros das Nações Unidas, o Brasil ocupou, em 2014, a 57a posição em e-government, ficando

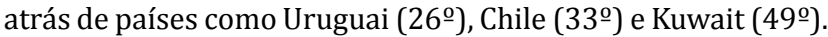
Considerando apenas os países da América, o Brasil ocupou a 8a posição, conforme mostra o quadro 2 .

Quadro 2. Top 20 em e-government nas Américas (Nações Unidas, 2014)

\begin{tabular}{|c|c|c|}
\hline Posição & País & EGDI \\
\hline 1 & Estados Unidos da América & 0.8748 \\
\hline 2 & Canadá & 0.8418 \\
\hline 3 & Uruguai & 0.7420 \\
\hline 4 & Chile & 0.7122 \\
\hline 5 & Argentina & 0.6306 \\
\hline 6 & Colômbia & 0.6173 \\
\hline 7 & Costa Rica & 0.6061 \\
\hline 8 & Brasil & 0.6008 \\
\hline 9 & Barbados & 0.5933 \\
\hline 10 & Antígua e Barbuda & 0.5927 \\
\hline 11 & México & 0.5733 \\
\hline 12 & Venezuela & 0.5564 \\
\hline 13 & Peru & 0.5435 \\
\hline 14 & Panamá & 0.5242 \\
\hline 15 & Grenada & 0.5220 \\
\hline 16 & Equador & 0.5053 \\
\hline 17 & El Salvador & 0.4989 \\
\hline 18 & São Cristóvão e Névis & 0.4980 \\
\hline 19 & Trinidade e Tobago & 0.4932 \\
\hline 20 & Bahamas & 0.4900 \\
\hline
\end{tabular}

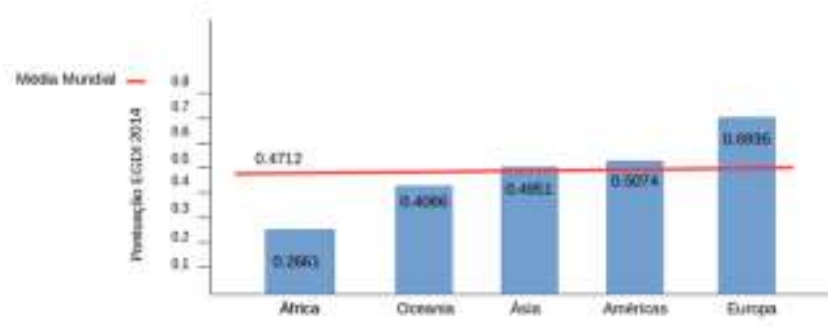

Figura 1. Médias continentais em desenvolvimento de e-government no ano de 2014 (Nações Unidas, 2014). 
Examinando pesquisas anteriores realizadas pelas Nações Unidas é possível perceber que o Brasil conseguiu atingir importantes posições, mas também significativas quedas. Em 2003, ocupava a $41^{\text {a }}$ posição, subindo para a 35을 posição no ano seguinte, passando por uma queda drástica em

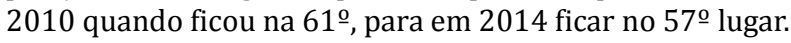

As Nações Unidas ainda medem a inciativa de governos em promover a abertura de dados governamentais (Open Government Data). Para isso, possui um instrumento intitulado E-Participation Index (EPI) que verifica se os governos (1) oferecem ferramentas de informação pública e acesso à informação sobre demanda - e-information-; (2) apresentam ferramentas de deliberação sobre políticas e serviços públicos - $e$-consultation; (3) capacitam pessoas na coprodução de componentes de serviços - e-decision-making.

Através desse instrumento, observa-se que o Brasil vem se mostrando equilibrado, ficando na $16^{\underline{a}}$ posição em 2003, e 24⿳亠口冋 posição em 2014. Todavia, em 2012 obteve uma queda, quando foi para o $42^{\circ}$ lugar. A figura 2 demonstra as evoluções e regressões do Brasil de acordo com os instrumentos de avaliação e-government e e-participation das Nações Unidas, de 2003 a 2014.

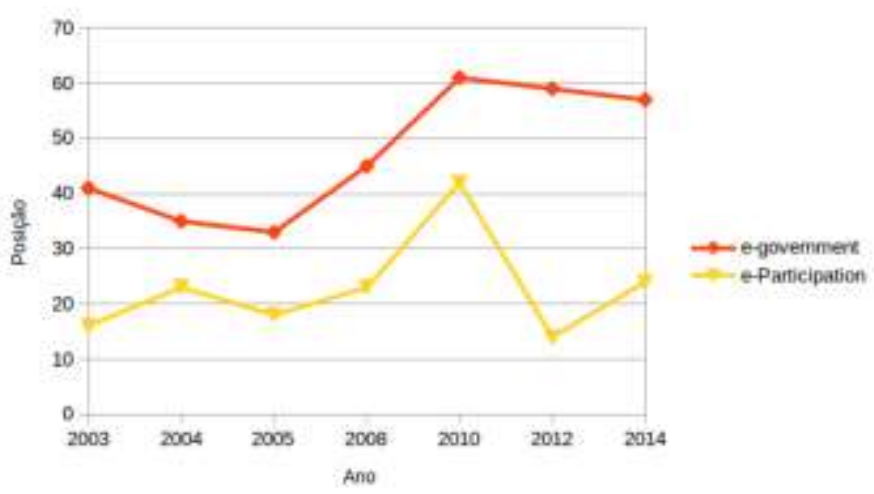

Figura 2: Evolução do Brasil em governo eletrônico de 2003 a 2014 (Fonte: elaborado pelo autor).

Uma das fontes de informação sobre o governo eletrônico brasileiro é o seu portal (http://governoeletronico.gov.br/). Nesse ambiente, encontram-se as diretrizes, políticas e práticas que orientam o governo federal no planejamento, especificação e desenvolvimento de iniciativas de e-government. Dentre as iniciativas do poder executivo federal, incluem-se os Padrões de Interoperabilidade (e-PING), Padrões Web (e-PWG), o Modelo de Acessibilidade em Governo Eletrônico (e-MAG), Dados Abertos Governamentais, Portal do Software Livre, Portal do Software Público, Portal das Comunidades Virtuais do Setor Público, Carta de Serviços ao Cidadão, entre outros.

Todavia, esse portal, mesmo tendo um aspecto centralizador, não apresenta todos os serviços prestados pelo governo, como a simplificação do processo de declaração do Imposto de Renda e o Portal da Transparência.

Além disso, o governo não aplica uma taxonomia clara sobre as iniciativas apresentadas, não indicando, por exemplo, quais tipos de serviço são voltados ao cidadão e quais são voltados a empresas, outros governos e servidores públicos.

É importante observar que além das ações do governo federal, há várias iniciativas em e-government em outros poderes (legislativo e judiciário) e nos outros níveis de governo (estadual e municipal). Propostas como o Portal ECidadania do Senado Federal, o Portal da Câmara dos Deputados $^{3}$, e o buscador LexML que organiza a informação legislativa e jurídica do país (Brasil, 2013a) são alguns dos exemplos.

Nos níveis estadual e municipal, os exemplos mais comuns são os portais online de notícias do governo, de transparência (como a apresentação de dados de folha de pagamento), de serviços prestados (como informações sobre veículos), e sistemas informatizados de matrículas escolares, de agendamento de consultas médicas e registro de ocorrência policial.

\section{Interoperabilidade}

A característica primária para que sistemas eletrônicos de governo existam é a adoção de Tecnologias da Informação e Comunicação (TIC). Isto porque, um grande número de atividades são suportadas por essas tecnologias, como na oferta de serviços e provimento de informações para que pessoas e organizações se conectem e interajam. Sobre o uso de componentes digitais, Pressman (2011) afirma que não há dúvida que uma porcentagem significativa de investimento será aplicado em produtos e serviços que tenham um componente digital - que são baseados em software ou controlados por software.

Considerando a importância do governo eletrônico, muitos países no mundo levam projetos dessa natureza como prioritários (Patnaik et al. 2008). E, por demandar soluções baseadas em componente digital, um projeto de governo eletrônico é também um projeto de desenvolvimento de software cujo sucesso depende de fatores que vão da definição de políticas e processos à qualificação de pessoal, investimento financeiro, estudo técnico, entre outros. Um dos requisitos fundamentais de um governo eletrônico é a necessidade de sua integração com unidades, internas e externas, de diversos sistemas, que em muitos casos funcionam com diferentes tecnologias. Dessa forma, aplicativos de governo eletrônico precisam integrar dados de diferentes unidades com diversos sistemas heterogêneos.

Nesse cenário, a interoperabilidade assume um importante papel para que as várias organizações parceiras se comuniquem sem a geração de dependência tecnológica entre elas. Segundo o Online Dictionary for Library and Information Science (ODLIS, 2013), o termo interoperabilidade refere-se "a capacidade de um sistema de hardware ou de software de se comunicar e trabalhar efetivamente no intercâmbio de dados com outro sistema, geralmente de tipo diferente, projetado e produzido por um fornecedor diferente".

Para Santos \& Reinhard (2012), a interoperabilidade é a capacidade de um sistema ou processo usar as informações e/ou a funcionalidade de outro sistema ou processo através da adesão a padrões comuns.

A definição apresentada por Machado et al. (2011) diz que interoperabilidade é a capacidade que sistemas e máquinas tem de trocar, processar e interpretar corretamente as informações, sendo condição essencial para a entrega aberta e flexível de serviços de governo eletrônico.

Segundo Farinelli et al. (2013), a interoperabilidade no governo eletrônico brasileiro se dá a partir da soma de vários esforços, como a integração de redes e a troca uniforme de dados, levando-se ainda em consideração a existência de diversas arquiteturas, plataformas de hardware e software.

Para Diniz et al. (2009), a interoperabilidade surge como elemento necessário à universalização de serviços do governo brasileiro, pois a infraestrutura dos serviços 
informatizados do governo federal foi fundada na operação de uma malha descentralizada, o que poderia comprometer o programa de diversas formas, seja pela falta de um padrão comum de atendimento, seja pela falta de intercomunicação, ou ainda pela ausência de procedimentos legais mais específicos.

Para atender essa demanda, o governo federal brasileiro lançou uma política orientada à construção de uma arquitetura de relacionamentos que envolvesse parceiros, fornecedores, cidadãos e outros governos.

Essa política culminou com a especificação da arquitetura Padrões de Interoperabilidade do Governo Eletrônico (e-PING), tendo como modelo o projeto e-GIF (Government Interoperability Framework) conduzido pelo governo britânico (Farinelli et al. 2013).

A primeira versão dessa arquitetura foi lançada em 2004 (Santos \& Reinhard, 2008), e ao longo dos anos foi revisada e reforçada por leis, decretos, portarias e instruções normativas. Exemplos incluem a Lei no 12.527/2011 (Lei de Acesso à Informação) ${ }^{4}$, a Lei n ${ }^{\circ} 12.965 / 2014$ (Marco Civil da Internet) ${ }^{5}$, e o Decreto nº 6.932/09 (Decreto Cidadão) que determina, entre outros itens, que órgãos do executivo federal deverão interoperar ao necessitarem de documentos comprobatórios de regularidade de situação do cidadão.

A arquitetura e-PING tornou-se um documento de referência, trazendo uma série de padrões que representam a estratégia do governo para o desenvolvimento de (1) novos sistemas de informação que vierem a ser desenvolvidos e implantados no governo federal e que se enquadram no escopo de interação, dentro do governo federal e com a sociedade em geral; (2) sistemas de informação legados que sejam objeto de implementações que envolvam provimento de serviços de governo eletrônico ou interação entre sistemas; e (3) aquisição ou atualização de equipamentos de TIC (BRASIL 2015a).

Segundo o Manual do Gestor de Interoperabilidade (Brasil, 2012a), diversas possibilidades são propiciadas pelos padrões de interoperabilidade adotados na e-PING, como escalabilidade das soluções, compartilhamento das informações entre os sistemas, respeito à autonomia dos sistemas envolvidos, simplicidade na construção de soluções, e possibilidade de trabalhar com sistemas heterogêneos (fornecedores, plataformas e tecnologias distintas).

Nesse sentido, a interoperabilidade gera instrumentos aos gestores para uma melhor tomada de decisão, uma melhor coordenação dos programas e serviços de governo e redução de custos, por meio da visão integrada dos processos do governo e do reúso de soluções tecnológicas (Brasil, 2012a).

A interoperabilidade traz ainda a possibilidade de se criar sistemas de "janela única". Sistemas desse tipo permitem a realização de buscas a recursos informacionais heterogêneos, em diferentes pontos, que ao serem reunidos dão ao usuário uma visão completa do recurso solicitado (Almeida, 2013). Logo, a concepção de sistemas de janela única implicam em um nível maior de integração, interligando sistemas diversos para facilitar o acesso a informações, em especial ao cidadão que em muitos casos se vê frente a uma oferta de serviços pulverizados.

Embora a interoperabilidade traga várias vantagens ela deve ser vista como um esforço de governança eletrônica bem pouco trivial em seus objetivos. Tornar sistemas de governo transparentes, no sentido de atuarem de forma independente, com baixo acoplamento, distribuídos, sobre diferentes plataformas, e ainda, observando o reúso e o intercâmbio de dados, demanda a definição de padrões que orientem essa prática e políticas de fomento que a sustentem.

Segundo Henning (2013), muitos governos falham em alcançar a interoperabilidade devido à falta de padrões e cumprimento de normas necessárias por parte dos integrantes de uma rede de colaboração. De acordo com Patnaik et al. (2008), apenas $15 \%$ dos projetos de e-governance foram implantados com sucesso ao passo que a maioria deles falharam ou ainda não mostraram os seus benefícios reais.

Santos e Reinhard (2012), ao realizaram uma pesquisa com aplicação de questionários a gestores de TI, pertencentes ao quadro de servidores da administração pública federal brasileira, constataram que mais de 33\% dos entrevistados apresentaram limitações e dificuldades para implementar as especificações da e-PING. Logo, o estudo concluiu que as limitações de ordem técnica e de qualificação profissional, além do desconhecimento do assunto ainda continuam como grandes barreiras para a adoção dos padrões de interoperabilidade no ambiente de governo eletrônico brasileiro.

Machado et al. (2011), em uma proposta similar, fizeram um estudo de caso da adoção da e-PING na Fundação Oswaldo Cruz (FIOCRUZ/Bahia). Sem entrar em detalhes técnicos sobre a ação de interoperabilidade, os autores procuraram discutir a e-PING como instrumento normatizador das ações de gestores de TI. Esse estudo constatou a resiliência por parte desses gestores a mudanças e a falta de capacitação técnica de pessoal da área de TI para a utilização de ferramentas relacionadas aos padrões adotados na e-PING.

Vários estudos apontam que a interoperabilidade desempenha um papel importante na definição de políticas para sistemas eletrônicos de governo. Nesse sentido, a arquitetura e-PING consolida a estratégia do governo federal brasileiro para implementações que envolvam provimento de serviços de governo eletrônico ou interação entre sistemas, dentro do governo e com a sociedade em geral.

Entretanto, pesquisas apontam para a baixa capacitação de profissionais da área de TI para lidar com um conjunto extenso e complexo de padrões de interoperabilidade, o que demandará uma possível política de formação. Dessa forma, entende-se que o problema de interoperabilidade com os atuais sistemas administrativos ainda é um grande desafio, mas os benefícios trazidos por ela fomentam e justificam iniciativas para a sua efetivação.

\section{Estudo de caso}

Nesta seção, são apresentados os resultados do estudo de caso realizado através da análise documental de projetos, leis, cartilhas, manuais e notícias coletados diretamente nos sítios web dos órgãos e entidades da administração pública federal. Para complementar, algumas publicações acadêmicas são citadas.

Em um primeiro momento, são apontadas algumas das iniciativas que compõem, no âmbito do poder executivo federal, a rede de serviços do governo eletrônico brasileiro. Nesse momento, é feita também uma discussão sobre as ações do governo eletrônico brasileiro destinadas à sociedade. Discute-se ainda, ações do governo que podem ser apropriadas pela comunidade de desenvolvedores de software, bem como as tecnologias adotadas pelo governo eletrônico para a integração de sistemas computacionais diversos.

Por fim, é feito o apontamento de aplicativos
A Lei de Acesso à Informação (Lei no 12.527/2011) regulamenta o direito constitucional de se obter informações públicas. Logo, todas as informações produzidas, ou sob guarda do poder público, são públicas e, portanto, acessíveis a todos os cidadãos, ressalvadas as informações pessoais e as hipóteses de sigilo legalmente estabelecidas.
O Marco Civil da Internet (Lei $\mathrm{n}^{\circ} 12.965 / 2014$ ) define como diretrizes para a atuação da União, dos estados, do Distrito Federal e dos municípios no desenvolvimento da internet, no Brasil, a promoção da racionalização e da interoperabilidade tecnológica dos serviços de governo eletrônico e a promoção da interoperabilidade entre sistemas e terminais diversos. 
computacionais que se integram a sistemas estruturantes do governo federal e aplicativos desenvolvidos pela comunidade que fazem uso de dados disponibilizados pelo governo eletrônico brasileiro.

\subsection{Iniciativas de Governo Eletrônico}

0 governo eletrônico brasileiro possui uma gama de serviços que se relacionam diretamente com as diretrizes que o sustentam. Alguns desses serviços estão relacionados à internalidade do governo, preocupando-se com a economicidade de recursos, otimização de ações, compartilhamento de dados e integração de serviços. Outras iniciativas se coadunam com os interesses da sociedade, principalmente pelo anseio popular por maior transparência das ações de governo - como a disponibilização de informações de caráter público (investimentos, pagamentos, transferência de valores, entre outros).

Dentre as iniciativas presentes na rede de serviços do governo eletrônico brasileiro, cujos conteúdos se direcionam ao cidadão (G2C), destacam-se a Inclusão Digital, o Portal do Software Público, o Portal do Software Livre, o Portal de Dados Abertos, o Sistema Eletrônico do Serviço de Informações ao Cidadão (e-SIC), o Sistema Protocolo Integrado, o Sistema de Informações Organizacionais do Governo Federal (SIORG cidadão), e o Portal da Transparência. Vale mencionar que esse último serviço não é citado diretamente no sítio web do governo eletrônico, mas surge como iniciativa mantida pelo governo federal. Essas iniciativas são discutidas a seguir.

A iniciativa intitulada Inclusão Digital atua em diferentes frentes, como na implantação de polos digitais, telecentros, centros de recondicionamento de computadores, e parcerias com municípios brasileiros para a criação de cidades digitais. Grande parte das ações dessa iniciativa são feitas via edital, seja para a contratação de serviços, para a seleção de municípios a serem contemplados com investimentos do governo federal, ou mesmo para a afirmação de parceria da União com os estados da federação. $\quad 0$ problema reside no fato de que esses editais se exauriram e não passam do ano de 2013, conforme publicado no sítio web do Ministério das Comunicações ${ }^{6}$. Por outro lado, foram feitas algumas inaugurações de cidades digitais, com fibra óptica para conectar órgãos públicos e pontos de acesso livre, em 2015. Logo, é possível inferir que esse é um projeto de governo que precisa ser revitalizado para melhor prover os programas de inclusão digital do governo federal.

O Portal do Software Público (www.softwarepublico.gov.br) conta atualmente com 69 softwares em seu catálogo. Esse portal criado em 2007, segundo o sítio web oficial, consolida um ambiente de compartilhamento de software que pode ser acessado por qualquer cidadão. Sua base de dados agrega um conjunto de soluções das mais variadas, como softwares para avaliar sistemas computacionais (nível de acessibilidade e performance), frameworks para desenvolvimento de aplicativos computacionais (exemplos, Cortex e Demoiselle) e sistemas de gestão e gerenciamento de processos. Para desenvolvedores de software, possui ainda uma ferramenta livre de gerenciamento de código fonte, intitulado GitLab.

A iniciativa de construir e manter um portal que agregue soluções de software é de salutar importância. Gestores de TI podem encontrar nesse instrumento soluções pontuais para as suas demandas. Governos podem reduzir custos ao verificarem que soluções similares publicadas satisfazem as suas necessidades. E a comunidade de desenvolvimento de software pode se sentir motivada a contribuir com essa iniciativa.

$$
0 \text { Portal do Software Livre }
$$

(www.softwarelivre.gov.br) atua em outra vertente. Nesse portal, há um conjunto significativo de informações que promovem ações de produção, divulgação e uso de software livre. Um item de destaque do portal é o espaço para a divulgação de experiências em migração e implementações de software livre. Trata-se de um catálogo de casos de sucesso com softwares livres que podem servir de referência para empresas e órgãos de governos. Embora atualmente possua apenas 12 relatos, o portal é uma importante ferramenta que poderia ser melhor explorada pelo governo e pela sociedade.

O Portal de Dados Abertos (www.dados.gov.br) é resultado de uma metodologia adotada pelo governo federal para a publicação de dados em formatos reutilizáveis. Esse portal, segundo Brasil (2015b), visa o aumento da transparência e maior participação política por parte do cidadão. Nesse portal, os dados catalogados estão organizados utilizando estruturas de conjuntos de dados e recursos. Informações das mais variadas podem ser consultadas, como dados da copa de 2014, ou mesmo dados de pesquisas realizadas pelo Instituto Brasileiro de Geografia e Estatística (IBGE).

O fato desse portal disponibilizar várias informações em formatos reutilizáveis contribui para uma participação mais ativa da sociedade ao permitir que o usuário faça as agregações e derivações que julgar mais interessante sobre dados em forma bruta. Todavia, o fator que deprecia essa iniciativa é a existência de recursos e conjunto de dados que direcionam a links inválidos.

Para o conjunto de dados do Portal de Dados Abertos, o governo eletrônico brasileiro disponibiliza aos desenvolvedores um manual, Brasil (2011), que oferece uma visão técnica sobre como publicar e reutilizar dados abertos. Esse manual parte da premissa que apenas colocar documentos na web não significa transparência pública, sendo preciso disponibilizar as informações de modo que humanos e máquinas consigam interpretá-las de forma ágil. Logo, oferece subsídios para estruturar web services, criar documentos em RDF (Resource Description Framework), web scrapping para varredura e coleta de dados de sítios web, entre outros.

O e-SIC representa uma demanda social por maior agilidade e presteza nas informações de caráter público. Através dele qualquer pessoa, física ou jurídica, pode requisitar e encaminhar pedidos de acesso à informação. No sítio web oficial do e-SIC (www.acessoainformacao.gov.br), há um manual que orienta o usuário sobre os procedimentos necessários para fazer uma solicitação.

Para desenvolvedores de software há um documento que define os serviços web (web services) do e-SIC (Brasil, $2013 \mathrm{~b}$ ) e um tutorial que demostra como consumir esses serviços utilizando a tecnologia Java (Brasil, 2012b). Notadamente, essa iniciativa harmoniza o desejo popular de poder acessar o conjunto de dados mantido pelo governo com o espírito democrático de uma sociedade participativa.

o Sistema de Protocolo Integrado (www.protocolointegrado.gov.br) tem como objetivo social oferecer ao cidadão uma visão integrada da tramitação de documentos. Atualmente, apenas o Ministério do Planejamento, Orçamento e Gestão e a Universidade Federal de Santa Maria enviam dados a esse sistema. Todavia, segundo a Portaria Interministerial № 2.320 , de 30 de dezembro de 2014, os demais órgãos da administração pública federal deverão adaptar os seus sistemas para integração até 2016. Em sua essência esse é também um importante instrumento já que visa ser um canal de buscas a recursos informacionais heterogêneos, armazenados em diferentes servidores na rede, através de interface única, em que o usuário não precise tomar conhecimento de onde nem como esses recursos estão armazenados ("janela única”). Para esse sistema há um manual técnico que visa auxiliar na utilização de seus serviços web 
(web services) (Brasil, 2015c). Contudo, desenvolvedores externos não podem acessar esses web services já que é necessário obter credenciais de acesso. $\quad 0 \quad$ SIORG cidadão (https://siorg.planejamento.gov.br) objetiva apresentar com maior transparência à sociedade a estrutura organizacional da administração pública federal (Brasil, 2014). Através dele é possível consultar as estruturas que compõem o governo, bem como gerar organogramas.

Para desenvolvedores há um documento de referência para se obter dados e serviços com uso de web services. Nesse documento, (Brasil, 2014a), há uma descrição detalhada de cada tipo de serviço disponibilizado pelo SIORG, todos estruturados com a arquitetura REST (Representational State Transfer).

Essa iniciativa do governo federal, embora importante, falha ao não trazer dados completos sobre as consultas realizadas. Por exemplo, essas consultas poderiam apresentar o nome, o telefone, e o e-mail de representantes das unidades organizacionais, como o próprio sistema sugere. Entretanto, a maioria das consultas retornam tabelas vazias.

$$
\text { o Portal da Transparência }
$$
(www.transparencia.gov.br) é um espaço de promoção de transparência pública. Nele é possível fazer consultas no âmbito do poder executivo federal, sobre receitas, despesas, convênios, servidores, entre outros. O padrão adotado nesse portal para downloads de arquivos é o Comma Separeted Values (CSV) e nenhum outro formato é utilizado. Logo, essa limitação pode influenciar negativamente a proposta já que gera uma dependência exclusiva a uma única tecnologia. Diferentemente de outras iniciativas aqui não se aplica o uso de web services.

Algumas iniciativas de importância para projetistas de sistemas, mais do que para a sociedade em sentido amplo, podem ser objetos de estudo e de aplicação. Os Indicadores e Métricas para Avaliação de e-serviços, (Brasil, 2007), constitui um importante instrumento que pode ser absorvido pela comunidade de desenvolvimento de software como modelo para a avaliação de serviços eletrônicos. Da mesma forma, o Modelo de Acessibilidade em Governo Eletrônico (e-MAG) pode ser levado em consideração no desenvolvimento de sistemas de informação pautados na acessibilidade (Brasil, 2014b). Outro documento importante é o Padrões Web em Governo Eletrônico (Brasil, 2012c) que traz recomendações de boas práticas com o objetivo de aprimorar a comunicação, o fornecimento de informações e serviços prestados por meios eletrônicos.

As iniciativas do governo eletrônico brasileiro, apresentadas nessa seção, criam possibilidades para a modernização do Estado, tornando-o mais participativo, transparente e efetivo. Neste contexto, buscou-se apresentar uma descrição sucinta sobre essas iniciativas, apontar instrumentos para uso do cidadão e por desenvolvedores de software, bem como discutir limitações.

Para atingir os resultados esperados desta etapa, foi necessário consultar documentos do governo eletrônico e sistemas desenvolvidos para a sua efetivação. Esse estudo de caso procurou, em especial, identificar fontes afirmativas para a participação da sociedade no exercício de sua cidadania. E através dele foi possível constatar que grande parte de documentos do governo eletrônico estão espalhados, demandando um vasculhamento das fontes e leitura minuciosa sobre eles, já que poderiam direcionar a outras fontes de interesse.

Através da análise documental realizada foi possível também perceber que alguns desses documentos fazem uso de endereços eletrônicos inválidos, mesmo àqueles com revisão recente. Por exemplo, o endereço www.eping.e.gov.br, apresentado na versão de 2015 da arquitetura e-PING, leva a um domínio inexistente.

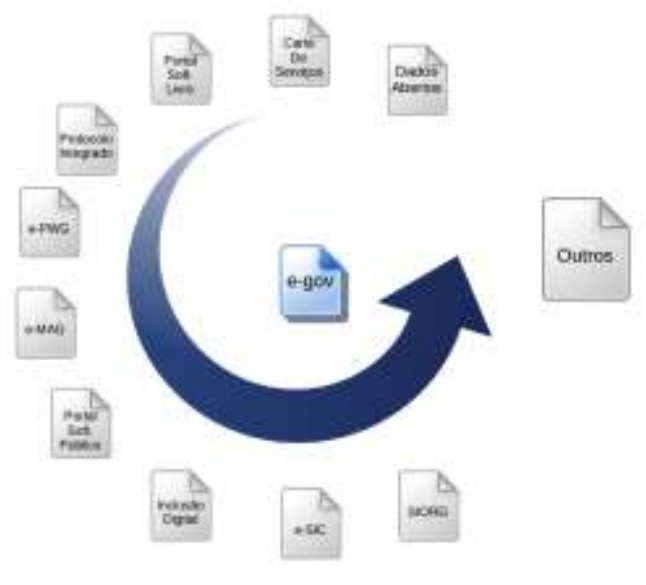

Figura 3. Iniciativas do governo eletrônico brasileiro (Fonte: elaborado pelo autor).

Da mesma forma, é possível perceber que a rede de iniciativas do governo eletrônico brasileiro acaba se tornando maior do que a apresentada em seu sítio web oficial. A figura 3 retrata alguns dos serviços que compõem o governo eletrônico brasileiro, mas enfatiza a existência de outras iniciativas.

\subsection{Iniciativas de Interoperação}

Com relação ao domínio técnico da interoperabilidade, o governo eletrônico brasileiro possui um documento de referência intitulado Padrões de Interoperabilidade do Governo Eletrônico (e-PING).

Esse documento, (Brasil, 2015a), orienta os órgãos da administração pública federal em suas soluções de interoperabilidade com a especificação de políticas gerais, como a adoção preferencial de padrões abertos, uso de software livre e/ou público, e soluções amplamente utilizadas pelo mercado.

Esta seção procura investigar soluções computacionais para a integração de sistemas de diferentes naturezas com os sistemas administrativos de governo, ao mesmo tempo em que identifica tecnologias para que essa integração ocorra.

Sobre o quesito organização e intercâmbio de informação, a e-PING adota a XML (Extensible Markup Language) e JSON (Javascript Object Notation) como linguagens para intercâmbio de dados. Adota ainda, o RDF (Resource Description Framework) para a descrição de recursos, a OWL (Web Ontology Language) como linguagem de definição de ontologias na web, e o SPARQL (Sparql Protocol and RDF Query Language) como linguagem de consulta semântica.

Entre outras especificações há a recomendação de uso da especificação UDDI (Universal Description, Discovery and Integration) como infraestrutura de registro, adoção do WSDL (Web Service Description Language) como linguagem de definição de serviço, e SOAP como protocolo para acesso a web services.

Diante dessa padronização, algumas propostas surgiram com o intuito de explorar esse conjunto de tecnologias adotadas pela e-PING. Lima et al. (2010) apresentam uma experiência desenvolvida pela Universidade Federal do Rio Grande do Norte (UFRN) para a integração e interoperação do sistemas da UFRN com os sistemas estruturantes do governo federal. Nesse trabalho, buscou-se formas de interação dos sistemas em uso pela UFRN com sistemas da União, entre eles: SIAFI (Sistema Integrado de Administração Financeira do Governo Federal), SIAPE (Sistema Integrado de Administração de Pessoal) e SIASGE (Sistema Integrado de Administração de Serviços Gerais). 
As soluções adotadas se resumem em grande parte pelo desenvolvimento de web services. Segundo os autores, essas soluções contribuíram para a eliminação de retrabalho e manutenção da consistência de dados, já que evitam o trabalho duplo de lançar dados tanto nos sistemas da UFRN quanto nos sistemas do governo federal.

Silva (2010) apresenta a solução e-STF - Processo Eletrônico Integração-, criada para a comunicação entre o Supremo Tribunal Federal e os demais órgãos do poder judiciário e da administração pública, tendo como objetivos transmitir peças e permitir a comunicação de atos processuais. Essa solução adota padrões de interoperabilidade recomendados pela e-PING, como web services para a comunicação e integração de sistemas heterogêneos através do protocolo SOAP. Segundo o autor, essa solução promove a melhoria do processo judicial e contribui para que a administração se modernize, estando mais próxima do cidadão e dando maior transparência e agilidade ao processo judicial.

Lotta et al. (2014) discutem a aplicabilidade do SIMEC - Sistema Integrado de Planejamento, Orçamento e Finanças do Ministério da Educação. Segundo os pesquisadores, esse sistema oferece impactos positivos enquanto ferramenta estratégica de gestão. De acordo com Cabral et al. (2010), o SIMEC utiliza várias ferramentas para possibilitar a interoperabilidade, dentre elas: web services, extração de arquivos e envio de arquivos XML via sockets. A integração desse sistema, segundo os autores, é feita com a Receita Federal, Correios, IBGE (Instituto Brasileiro de Geografia e Estatística), SIGPLAN (Sistema de Informações Gerenciais e Planejamento), SIDOR (Sistema Integrado de Dados Orçamentários), SIAFI e SIAPE.

As iniciativas de integração não se limitam a ações de governo. A sociedade também vem participando desse processo através do desenvolvimento de aplicativos computacionais. O Portal de Dados Abertos reúne, até o momento, um catálogo com onze soluções desenvolvidas pela comunidade. Um exemplo dessa iniciativa popular é o aplicativo Reclamações Procon (figura 4), nome sugestivo para um sistema que traz informações com visualização de gráficos e figuras sobre reclamações registradas, no ano de 2011, no Programa de Proteção e Defesa do Consumidor (PROCON). Outro exemplo, é o aplicativo Aeroportos Brasil que mostra o movimento de aeronaves e passageiros nos aeroportos administrados pela Empresa Brasileira de Infraestrutura Aeroportuária (Infraero).

Essa seção apresentou uma revisão sumária sobre o assunto tema, portanto, não é estanque, visto que várias outras iniciativas devem estar em curso. Em parte, iniciativas que objetivam explorar as potencialidades do governo eletrônico brasileiro, em especial, a arquitetura e-PING.

Nesse sentido, a abordagem empregada nesse trabalho amplia a discussão ao dar luz às possibilidades que podem ser feitas por agentes de inovação na produção de sistemas interoperáveis. Além disso, ao apontar tecnologias adotadas pelo governo para aplicar a interoperabilidade entre sistemas, fornece a projetistas de software um conjunto de informações que devem ser investigadas, inclusive no desenvolvimento de sistemas que se comuniquem com as iniciativas discutidas na seção anterior.

\section{Conclusão}

O governo federal brasileiro possui um conjunto de ações que fortalecem e efetivam a existência do governo eletrônico. Essas ações vão da publicação de dados para consumo pela sociedade à organização e gerenciamento interno dos sistemas estruturantes de governo. Dentre essas ações, a atenção dada à efetivação da participação cidadã é um elemento chave na promoção da cidadania, já que a sociedade passa a possuir instrumentos para se manifestar junto às ações governamentais.

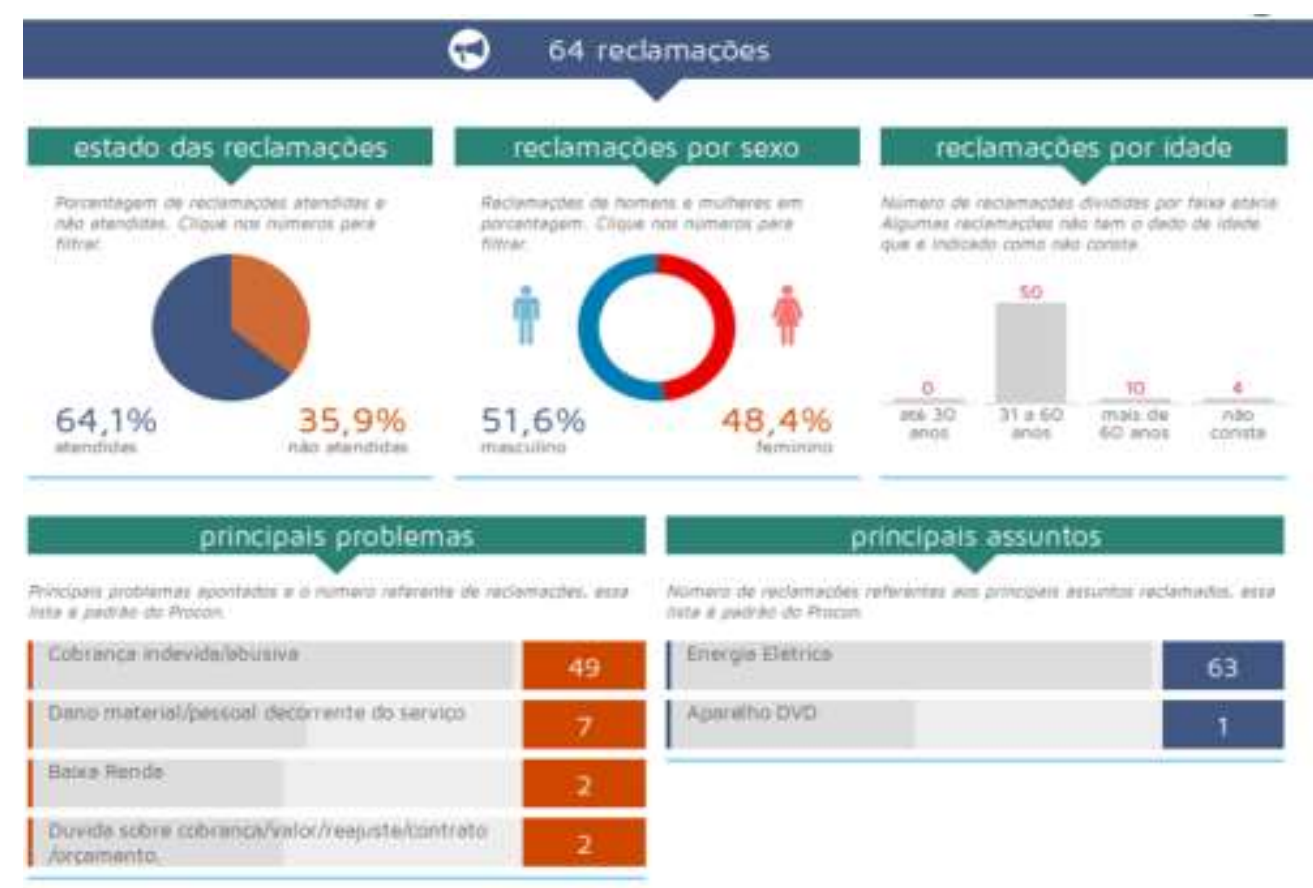

Figura 4. Aplicativo Reclamações Procon (http://www.reclamacoesprocon.com.br/)

Ao longo de quinze anos de amadurecimento, as iniciativas promovidas pelo governo eletrônico brasileiro vão aos poucos tomando forma e sendo apropriadas pela sociedade. Essas iniciativas então passam a se tornar direitos que extravasam uma política de governo e aos poucos assumem um caráter de política de estado. A tendência é que novas iniciativas surjam e coloquem a sociedade mais próxima dos representantes que ela mesma elegeu.

Para a afirmação de um governo eletrônico o componente mestre é a sua arquitetura. Através dela, forma-se uma rede integrada que racionaliza recursos em tecnologia da informação e comunicação. Além disso, ela sustenta uma 
política de reutilização, bem como ações de implantação e operação de uma série de fatores críticos. No caso do governo eletrônico brasileiro, a e-PING é o modelo de referência arquitetural. Esse modelo utiliza um arcabouço de padrões tecnológicos para diferentes níveis no tratamento da informação, que possibilita a consistência e clareza na implementação de platôs de infraestrutura. Nesse contexto, web services, e as tecnologias que os compõem, aparecem como importantes agentes.

Embora as iniciativas do governo eletrônico brasileiro sejam válidas, em alguns casos os serviços que o compõem apresentam problemas que vão desde a colocação de informações dispersas entre vários sítios - o que demanda "garimpar" as informações -, até a indicação de links inválidos e/ou indisponíveis. Poderia então, para facilitar o entendimento, preparar uma revisão sobre os seus serviços, organizando-os de acordo com a taxonomia G2G (government to government), G2B (governments to businesse), G2C (government to citizens), e G2E (government do employee). Todavia, é possível identificar um conjunto significativo de instrumentos que orienta o cidadão na utilização desses serviços, incluindo tutoriais e manuais que se direcionam à comunidade de desenvolvimento de software.

Por fim, observa-se ainda que falta ao governo divulgar sua plataforma eletrônica com mais consistência, bem como estimular a sociedade na propositura de ações de integração com dados governamentais. Nesse sentido, o Portal do Software Público poderia possuir um catálogo maior de soluções em sistemas de informação. 0 Portal do Software Livre poderia apresentar mais relatos de experiência em migração e desenvolvimento de software livre. O Portal de Dados Abertos poderia ter um número mais representativo de aplicativos desenvolvidos pela comunidade para consumir os dados disponibilizados. E, ações de formação técnica deveriam ser tomadas com maior ênfase, para preparar tanto gestores de TI como a sociedade para utilizar com maior propriedade $o$ conjunto amplo de padrões adotadas na e-PING.

\section{Referências}

Almeida, Djalma Fiuza. (2013). Governança Pública, Interoperabilidade e Interoperatividade: Desafios para a Gestão do Dado Institucional na UNEB. (Dissertação de mestrado) Universidade do Estado da Bahia, Brasil.

Alshehri, M., Drew, Steve J. (2011). E-government principles: implementation, advantages and challenges. International Journal of Electronic Business, 9(3), 255270.

Brasil. (2000). Portaria n. 23, de 12 de maio de 2000. Institui Grupo de Trabalho Interministerial. Disponível em $<$ www.governoeletronico.gov.br/biblioteca/arquivos/p ortaria-da-casa-civil-no-23-de-12-de-maio-de2000/view>. Acesso em: 08/06/ 2015.

(2007). Indicadores e Métricas para avaliação de eServiços. MPOG. SLTI. Departamento de Governo Eletrônico. Brasília: MP.

(2009). Decreto $n$. 6.932. Disponível em <http://www.planalto.gov.br/ccivil_03/_ato20072010/2009/decreto/d6932.htm> Acesso em: $07 / 07 / 2015$.

(2011). Lei n. 12.572. Disponível em $<$ http://www.planalto.gov.br/ccivil_03/_ato20112014/2011/lei/l12527.htm> Acesso em: 02/08/ 2015. . (2011). Manual dos Dados Abertos: desenvolvedores. São Paulo: Comitê Gestor da Internet no Brasil.

. (2012a). Guia de Interoperabilidade: Manual do Gestor. Brasília: MP.

(2012b). e-Sic: Tutorial Consumindo Webservices Java, versão 1.1. Disponível em: <http://www.acessoainformacao.gov.br/webservices>.
Acesso em: 01/07/2015.

. (2012c). e-PWG: Padrões Web em Governo Eletrônico. MPOG. SLTI. Departamento de Governo Eletrônico. Brasília: MP.

. (2013a). Senado Federal. Manual de pesquisa do Portal LexML. Brasília: Senado Federal.

. (2013b). e-Sic: Definição dos Webservices, versão 1.4 . Disponível

em: <http://www.acessoainformacao.gov.br/webservices>. Acesso em: 01/07/2015.

. (2014a). Documentação do Novo Web Service SIORG, versão 1.1. Brasília: MP, SEGEP.

. (2014b). e-MAG: Modelo de Acessibilidade em Governo Eletrônico. MPOG. SLTI. Departamento de Governo Eletrônico. Brasília: MP.

(2014) Lei n. 12.965. Disponível em <http://www.planalto.gov.br/ccivil 03/ ato20112014/2014/lei/l12965.htm> Acesso em: 12/08/2015. . (2014). Portaria Interministerial n. 2.320. Disponível em <http://www.comprasgovernamentais.gov.br/paginas/ portarias/portaria-interministerial-no-2-320-de-30-dedezembro-de-2014> Acesso em: 30/08/2015.

(2015a). e-PING: Padrões de Interoperabilidade de Governo Eletrônico. MPOG. SLTI. Departamento de Governo Eletrônico. Brasília: MP.

. (2015b). Dados Abertos Governamentais. Disponível em: $<$ https://www.governoeletronico.gov.br/acoes-eprojetos/Dados-Abertos > Acesso em: 06/07/2015.

(2015c). Sistema Protocolo Integrado: Manual de Integração ao Web Service. Brasília: MP,.

Cabral, C. Bueno, J. E., Sousa, D. B. (2010). SIMEC: uma mudança na cultura de gestão. In Mesquita, Claúdia S. F., Bretas, Nazaré L. (Orgs), Panorama da interoperabilidade no Brasil (pp. 201-210). Brasília: MP.

Crowley, Megan. (2008). E-government: an introduction. Center for Public Policy \& Administration, 4(6), Disponível em:

$<\underline{\text { http://www.imakenews.com/cppa/index000274284.c }}$ $\mathrm{fm} ? \mathrm{x}=\mathrm{b} 11,0, \mathrm{w}>$ Acesso em: 20/08/2015.

Diniz, Eduardo H., Barbosa, A. F., Junqueira, A. R., Prado, 0. (2009). O governo eletrônico no Brasil: perspectiva histórica a partir de um modelo estruturado de análise. Revista de Administração Pública, RIO DE JANEIRO 43(1), 23-48.

Farinelli, Fernanda, Melo S., Almeida, M. B. (2013). O papel das ontologias na interoperabilidade de sistemas de informação: reflexões na esfera governamental. In XIV Encontro Nacional de Pesquisa em Ciência da Informação, Santa Catarina, Brasil, 29 de out. a 01 de nov. 2013 (pp. 1-21).

Henning, F. (2013). Adoption of interoperability standards in government information networks: an initial framework of influence factors. In Proceedings of the 7th International Conference on Theory and Practice of Electronic Governance, 2013, (pp. 264-267).

Laia, Marconi M., Cunha, Maria. A. V. C., Nogueira, Antonio R. R. N., Mazzon, José A. (2011). Electronic government policies in Brazil: context, ICT management and outcomes. Revista de Administração de Empresas, 51(1), 043-057.

Lima, G., West, M., Beltrão, P. Galhardo, R. (2010). Interoperabilidade do InfraSIG - UFRN/MJ. In Mesquita, Claúdia S. F., Bretas, Nazaré L. (Orgs), Panorama da interoperabilidade no Brasil (pp. 176-193). Brasília: MP.

Lotta, G. S., Farias, G. S., Ribeiro, E. R. (2014). Sistema integrado de monitoramento, execução e controle (SIMEC): usos e impactos na cultura administrativa no município de São Bernardo do Campo. Revista Temas de Administração Pública, 9(2). 
Machado, Kaliane, Júnior, A., Santos, E. M. (2011). A Adoção da Arquitetura e-PING: Um Estudo de Caso na FIOCRUZ/BAHIA. In III Encontro de Administração da Informação, Porto Alegre, Brasil, 15-17 set. 2011 (pp. 113).

Misra, Dinesh C. (2006). Defining e-government: a citizencentric criteria-based approach. In 10th National Conference on e-Governance, Bhopal, Madhya Pradesh, India, 01-03 fev. 2006 (pp. 1-11).

Nações Unidas. (2014). E-Government Survey 2014: $e$ government for the future we want. Disponível em: $<$ http://unpan3.un.org/egovkb/en-us/Global-Survey> Acesso em: 15/08/2015.

Obeidat, Rand A., Abu-Shanab, Emad A. (2010). Drivers of EGovernment and E-Business in Jordan. In Journal of Emerging Technologies in Web Intelligence, 2(3), 204211.

ODLIS. (2013). Online Dictionary For Library And Information Science. Disponível em: <www.abcclio.com/ODLIS/odlis i.aspx>. Acesso em: 02/06/2015.

Patnaik, P., Krushna, R., Patra, Manas R. (2008). Key Characteristics of Indian e-Governance Projects: A Special Reference to "Bhoomi". E-governance in Practice, SIGeGOV Publications, 33-41.

Pressman, Roger S. (2011). Engenharia de Software: uma

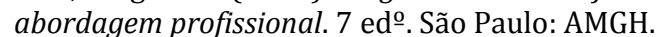

Santos, Ernani M., Reinhard, Nicolau. (2008). Interoperability standards for electronic government in Brazil: an exploratory study of the Implications of the e-ping framework implementation. In 5th International Conference on Information Systems and Technology Management, São Paulo, Brasil, 04-06 jun. 2008 (pp. 4126-4141).

Santos, Ernani M., Reinhard, Nicolau. (2012). Electronic Government Interoperability Identifying the Barriers for Frameworks Adoption. Social Science Computer Review, 30, 71-82.

Shailendra, C., Palvia, J., Sharma, S. (2007). E-Government and E-Governance: Definitions/Domain Framework and Status around the World. In Proceedings of the $5^{\text {th }}$ International Conference on e-governance, Hyderabade, India, 20-30 dez. 2007 (pp. 1-12).

Silva, Lúcio M. (2010). e-STF Processo Eletrônico: Integração. In Mesquita, Claúdia S. F., Bretas, Nazaré L. (Orgs), Panorama da interoperabilidade no Brasil (pp. 194-200). Brasília: MP.

Tang, C., Perumal, M. R. (2013). The Characteristics and Values of E-governance and the Role of E-democracy. International Journal of Humanities and Management Sciences (IJHMS), 1(1), 142-145.

Tonhati, Tania. (2011). Governo Eletrônico, Globalização e perspectivas da gestão pública para a democracia digital. Revista Democracia Digital e Governo Eletrônico, 5, 1-13. 